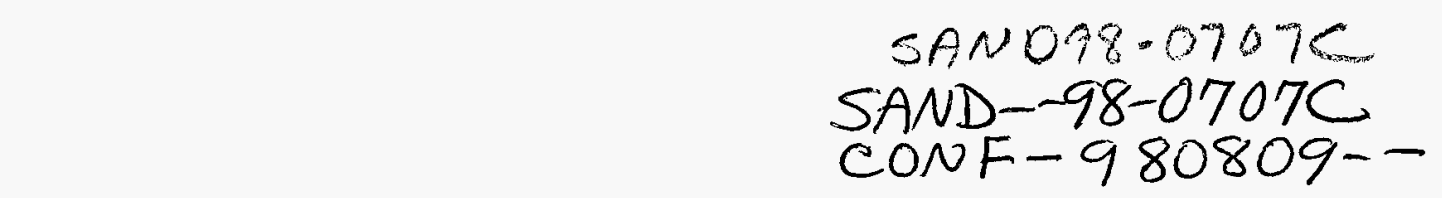

\title{
Risk Assessment and Integrity in System Design ${ }^{1}$
}

\author{
Ray S. Berg and Victor Winter \\ Sandia National Laboratories \\ rsberg@sandia.gov and vlwinte@sandia.gov
}

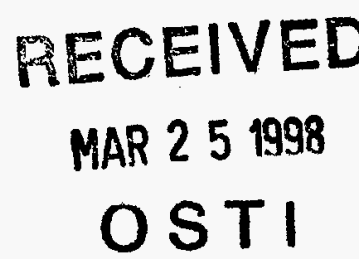

\begin{abstract}
All systems, regardless of how carefully they have been constructed, suffer failures. This paper focuses on developing a formal understanding of failure with respect to system implementations. Furthermore, we would like the system design process to be able to leverage off of this understanding. It is important to deal with failures in a system context, rather than a priori limiting the solution to a particular technology, such as software alone. Our approach is limited to the class of systems that can be modeled by hybrid finite state machines (HFSMs) as described in Winter [1].
\end{abstract}

The purpose of this paper is to lay out a process, or framework, that can aid in identification and characterization of techniques for dealing with the different types of system threats. This framework leads naturally to a taxonomy of technologies and strategies for dealing with the various types of threats. In this process technologies are used to identify a priority list of technical capabilities for dealing with threats. The technologies are prioritized according to their analyzability and predictability. Strategies are then used to identify specific implementations that are best suited to dealing with the threat

\section{Introduction}

Historically, the use of formal methods and techniques have been employed to improve the correctness of system designs and, ultimately, their implementations. However, in any system implementation there will be failures that result from our inability to produce perfect systems. In high consequence systems, there is a need to provide protection against the effects of high negative impact system failures. Such failures can harm the public, damage costly equipment, damage financial and other vital national institutions, and even destroy reputations.

We define a hazard as some aspect of a system that threatens the system or that has the potential for leading the system into a failure state. From a high-level perspective, there are two sources of such threats in system implementations. The first type of threat arises from the fact that the states and transitions in a concrete system reflect the imperfect ability of our system to realize a specified behavior within its environment. These imperfections manifest themselves in the ability of the system to do more than is intended. An ideal system performs those functions, and only those functions, that are specified by the system requirements definition. However, the implementation of a system often has the ability to be ordered by the system controller to enter failure states or other states that are not part of the system definition.

The second type of threat arises from altered system functionality that results from aging or other environmental stresses within the system's environment. For example, such alterations can arise from the failure of the hardware on which software must run. Techniques for dealing with this type of threat cannot generally be applied until concrete implementations have been proposed, since the threats are a consequence of the implementation itself.

Ultimately, any form of system failure regardless of its source is undesirable. However, it can be useful to classify the source of a potential failure, or threat, because the methods for identifying, understanding and mitigating system threats can be different depending on the source of the threat. Most importantly, though, the threat is associated with a state of the system, and it is the transition from the state to a failure that must be defended against.

\section{System Model}

\subsection{System definitions}

Reactive Systems: The systems discussed in this paper are reactive systems, in the sense defined by Winter [1]. In that model, systems can be defined in terms of

\footnotetext{
${ }^{1}$ This work was supported by the United States Department of Energy under Contract DE-AC04-94AL85000. Sandia is a multi-program laboratory operated by Sandia Corporation, a Lockheed Martin Company, for the United States Department of Energy.
} 


\section{DISCLAIMER}

This report was prepared as an account of work sponsored by an agency of the United States Government. Neither the United States Government nor any agency thereof, nor any of their employees, makes any warranty, express or implied, or assumes any legal liability or responsibility for the accuracy, completeness, or usefulness of any information, apparatus, product, or process disclosed, or represents that its use would not infringe privately owned rights. Reference herein to any specific commercial product, process, or service by trade name, trademark, manufacturer, or otherwise does not necessarily constitute or imply its endorsement, recommendation, or favoring by the United States Government or any agency thereof. The views and opinions of authors expressed herein do not necessarily state or reflect those of the United States Government or any agency thereof. 
environmental state changes that occur in the physical world of the system. Specific state changes result from a combination of the state of the system and event initiators that trigger the state change. Reactive systems can be described in terms of a collection of control and monitor variables. The monitor variables represent values generally supplied by various sensors that describe the system state. If the system were perfect, the system monitor variables would define the state of the system. The control variables represent values that are used to produce various system behaviors, such as operation of valves, alarms, and other devices. Thus, the function of a controller is to 1) use the information provided by the monitor variables to determine the state of the system, and 2) adjust the values of the control variables in order to achieve a desired system state or behavior. Thus, an ideal system is described by the equation:

$$
\underline{\mathrm{m}}^{\prime}=\mathrm{F}(\underline{\mathrm{m}}, \underline{\mathrm{c}})
$$

where $m$ ' is the vector of new monitor variables, $\underline{m}$ is the original vector of monitor variables, and $\underline{\mathbf{c}}$ is the vector of control variables. Since the controller can change the state of the system, it is considered to be an agent of the system.

Since the environment has the ability to change the state of the system, it is also considered to be an agent of that system. Thus, the systems described here are generally multiple agent systems. In this case we can consider a vector of virtual monitor variables, $\underline{m}_{e}$, and a vector of virtual control variables, $\underline{c}_{e}$, that are the result of the environment. The system then is described by the equation:

$$
\left\{\underline{m}^{\prime}, \underline{m}^{\prime}{ }_{e}\right\}=\mathrm{G}\left(\left\{\underline{\mathrm{m}}, \underline{\mathrm{m}}_{\mathrm{e}}\right\},\left\{\underline{\mathrm{c}}, \underline{\mathrm{c}}_{\mathrm{e}}\right\}\right)
$$

where $\left\{\underline{m}^{\prime}, \underline{m}_{e}\right\}$ is the new vector of monitor variables, $\left\{\underline{m}, \underline{m}_{\mathrm{e}}\right\}$ is the original vector of monitor variables, and $\left\{\underline{\mathbf{c}}, \underline{\mathbf{c}}_{e}\right\}$ is the vector of control variables. The use of this type of model has broad implications for the application of formal methods for system correctness by design.

State Machine Model: A system can be described in terms of a combination of an "ideal" system, which has only those states and transitions required for correct operation, and artifacts of "real" concrete implementations, which are those states and transitions that are an undesirable consequence of a specific implementation. The latter reflect our inability to design and build perfect systems. They include 1) additional states introduced by concrete implementations that have unintended or undesired features, and 2) effects in the system environment that initiate transitions to failure states. Figure 1 shows a representation of such a system.
In this system, states labeled with "S" are normal states that are invoked by the system controller. However, states labeled with " $F$ " are undesired consequences of the implementation. Some undesirable states, such as F2 and F3, may result in complete failures, such as the system locking up. Other failure states, such as F1, may be merely a nuisance to the user in that they result from added functionality that was not required of the system.

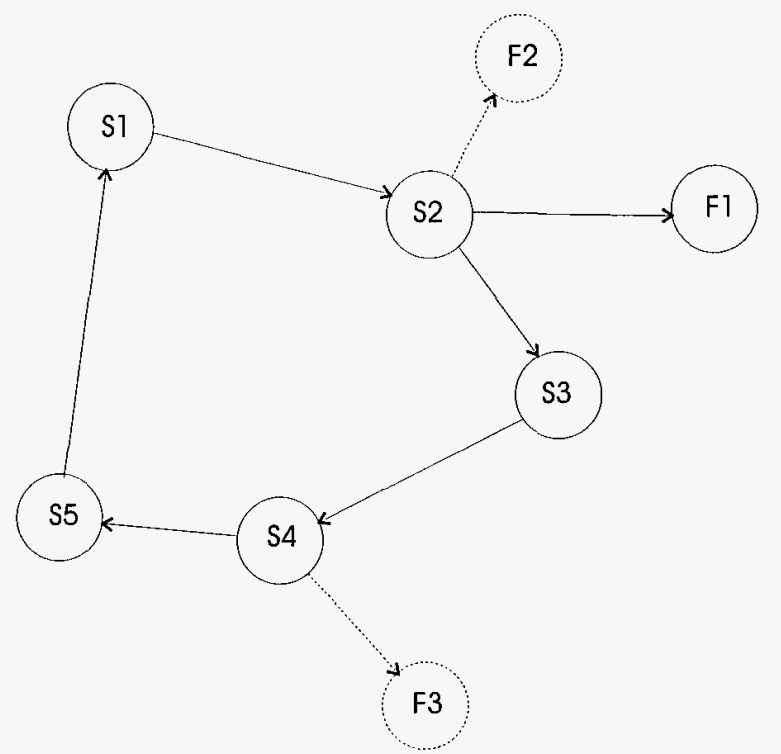

Figure 1. A state representation of a system.

\subsection{Systems based on event initiators}

If there are flaws in the design or undesirable functions introduced by the software or hardware, the controller may be able to initiate system transitions that lead to the undesirable or failure states. Other system failures may be the result of system hardware failures or software errors that either initiate or contribute to the initiation of transitions of the system that can lead to a failure state. In the worst case, the system may be unable to escape from such a failure state.

Each state transition can be expressed mathematically as the result of the initial state of the system and the event initiators that can cause the system to transition to a new state, as shown in the first equation above. The representation of the event initiators may include information about the function that produces the transition, the implementation technology, and the risks associated with the transition. This expansion allows explicit examination of choices of strategies and technologies to handle the associated threat, as will be discussed later.

\subsection{Failure Event Initiators}


Failure State Sources: The differences among design flaws, undesirable add-on functionality, and system failures are primarily in their origins rather than their effects. In order to deal with such defects as efficiently as possible, it is desirable to detect and deal with them as early in the design cycle as possible. Therefore, it is important to understand their origins. Unfortunately, in may cases their origin remains hidden, and it is not possible to identify it. Rather, it is only possible to note that the problem (i.e., failure) can occur and to provide a mechanism for defending against it or for mitigating its effects. The sequence of events is illustrated in Figure 2. It consists of 1) an initiating event, I, that causes a hazard and 2) a controller directed transition, $\mathrm{T}$, to a state, $\mathrm{S} 2$, that can interact with the hazard, followed by 3 ) an environmental or controller directed transition that the hazard causes to be directed to a failure state, F1.

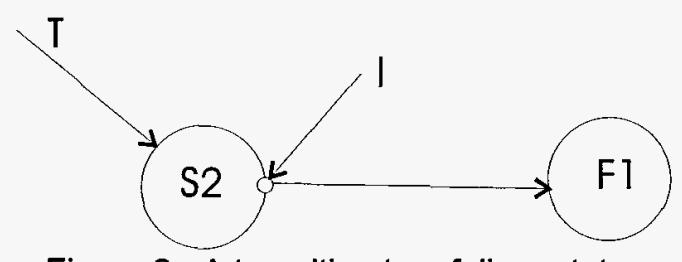

Figure 2. A transition to a failure state.

Defense usually involves reducing the probabilities of the initiating event or the transition, or placing more states between the normal system operation and the failure state, which has a similar effect.

Risk: Risk is the combination of the probability of occurrence of a specific event (initiator, I) and the consequences of that event (transition to failure state F1). In many current high consequence systems, the consequences of a system failure, at whatever probability of occurrence, is the dominant concern. Examples of such extreme consequences include unauthorized use of nuclear weapons, inability to shut down a nuclear reactor or chemical processing plant, and numerous others.

Protection cannot adequately be built around mitigating the consequences of such a high consequence failure. Therefore, it is important to understand the relationship between a transition to a failure state and its initiating event or events.

With the modern formal methods currently in use, the incidence of undesirable states is becoming significantly lower. However, we will never be able to build perfect systems, i.e., systems that will not suffer any failures, such as hardware failures. Traditionally, the treatment of failure has involved techniques of fault tolerance. But for very low probabilities of occurrence, say below about 1e6 , it is neither practical nor realistic to attempt to verify them experimentally. Even estimates of such events are difficult if not impossible due to their assumptions.
Indeed, some critics of current safety practices have suggested that the only valid assumption for such an initiating event is that if it can occur from a given state, we must assume that it will.

A defense against failures requires that we identify the specific states from which transitions to high consequence failure states can be triggered, and to provide protection against the occurrence of their initiating events. This will not involve all of the system's states, since many states, even in high consequence systems, have a rather mundane purpose and function. This can place a significant burden on the system designer to identify those states that are sensitive to high consequence failure so that defense against potential transitions to failure states can be incorporated into the system design.

\section{Notion of Integrity}

\subsection{Underlying Process}

Ekman [2] refers to a process in which a high consequence system design for safety must be capable of demonstrating predictable safety. Predictable safety is not based on traditional engineering "black box" design and interface testing. Rather, it is a methodology that relies the use of techniques that are analyzable and predictable for defending against specific undesired state transitions.

Integrity: Integrity refers to the ability of the system to perform functionally despite hazards that threaten the system's operation. While a design function is derived from a functional requirement, an integrity function is derived from an integrity hazard that threatens the system. There are two elements to the notion of an integrity requirement. The first element is the technology used to address the hazard. Our confidence in the reliability of a particular implementation is the result of our understanding of it, i.e., its analyzability and predictability. A simple mechanical device is generally much more analyzable and predictable than a software program. A general integrity mitigation and defense taxonomy must include a hierarchy of technologies that cover a spectrum of analyzable and predictable implementations. Those that are more analyzable and predictable in this context are generally preferred in system applications that are defending against transitions to high consequence failure states. For example, they may be crucial elements in a fail safe state transition.

The second element is the strategy used to deal with the threat. A particular strategy is only useful in the context of the threat with which it is dealing. For example, defense against a potential single point hardware failure might use multiple channel redundancy, 
such as a 3 of 4 voting scheme to reduce the probability of a transition to a failure state. However, that strategy would be useless against a potential common mode hardware failure, such as power source failure. A general integrity mitigation and defense taxonomy must also include an expansion of techniques that covers a spectrum of strategies that are capable of dealing with all credible failures. Thus, we also need a list of strategies that can handle specific types of failures.

The technology hierarchy orders technology classes according to their analyzability and predictability, while the strategy list identifies strategy classes that are applicable within the various technologies to handling a spectrum of threats. The technology list and strategy classes form a two dimensional integrity hierarchy, as illustrated diagramatically in Figure 3. Our mitigation and defense taxonomy should ideally link the two. For example, if you could conclude that a controller was designed correctly and that a particular state would fail in a benign manner, then you would not need to design in system defenses against such a controller failure. Similarly, if the system can defend itself from evil controller commands, then we also might not need to worry much about designing in defenses against such improper controller commands. Technology lists and strategies are discussed in more detail below.

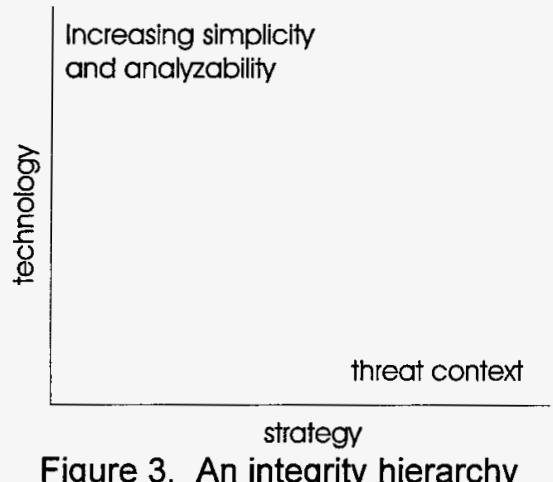

\subsection{A Technology Hierarchy -- An Integrity Technology Taxonomy}

A generic integrity taxonomy for dealing with integrity hazards must be able to deal with several types of failures. The highest level taxonomy consists of a technology hierarchy that encompass a spectrum of predictability and analyzability. In general, the more predictable and analyzable a technology is, the more desirable it is in high consequence systems. Dunn [3] developed a technology hierarchy of design guidelines for handling high consequence activities. This example includes the following:
1. physical barriers (e.g. mechanical stops)

2. static mechanical devices (e.g. burst disks)

3. Moving mechanical devices (e.g. relief valves, check valves)

4. Simple electromechanical systems (e.g. limit switches, pressure switches)

5. Simple analog or discrete digital electronic systems (e.g. overspeed circuits)

6. Simple software based devices (e.g. programmable logic controllers)

7. Redundancy (e.g. triple modular redundancy, standby sparing)

The implications of such a list are significant. In many modern systems software is assigned the responsibility for virtually all activities. However, many excellent, simple hardware protection devices can be included in the system when an integrated system approach is used.

\subsection{Strategy Classes -- An Integrity Strategy Taxonomy}

Similarly, the integrity taxonomy must discuss strategies that can be utilized to defend against specific system failures. There are several general classes of integrity strategies that can be used to help contain failures. Examples of high level taxonomies that deal with failures includes the following:

1. Generic: redundancy, diversity, defense-in-depth, fail safe, fault management

2. Software: life cycle management, tools, metrics, firewalls

3. Hardware: life cycle management, tools, metrics, security

4. Human Performance: cognitive behavior, training, simulation, security

5. Nuclear Ordinance: independence, incompatibility, inoperability, isolation

Each of these classes can be expanded into more detailed taxonomies of strategies to deal with specific kinds of failures. An example of such a taxonomy for diversity and defense-in-depth of hardware and software systems is shown in [4]. Once technologies and strategies are identified for defending against specific transitions, their features can be incorporated into the system design.

\subsection{Integrity Technology-Strategy Examples: failures, successes and serendipity}

We have described a methodology where systems are modeled in terms of HFSMs and transitions between states are constrained by event initiators. Technologies that are associated with event initiators at one level of abstraction can be modeled by their own HFSM at a 
lower level of abstraction. The purpose of this framework is to formalize a system design methodology that structures and makes explicit the aspects of system design that mitigate and/or defend against the occurrence of integrity hazard induced failures. Integrity hazards are undesirable events that can cause loss of function of a system that responds, directly or indirectly, to a system failure [5]. In response to the search for such strategies and technologies, several types of integrity taxonomies have been developed; for example, reference [4]. Such strategies can then be included in the design to deal with a failure when it occurs. Some examples of a technologystrategy taxonomy are useful as an illustration.

\subsubsection{Imperfect system functionality: design flaws.}

Earlier we discussed ideal systems as those that those features, and only those features, that were specified by the system requirements. Design flaws occurred because the implementation of the system introduced states that had undesirable side effects. Thus, design flaws result in the ability of the system controller to initiate transitions of the system's state that lead to an unacceptable or failure state from which it may not be able to recover. An example of this type of failure is the use of software to prescribe and enforce a design envelope in the F-18. The "fly-by-wire" F-18 military aircraft exhibited such a failure when the software allowed the aircraft to be placed into an attitude from which the software would subsequently not allow the pilot to escape [6]. Reports of the investigation of F-18 crashes initially attributed to pilot error found they were caused by programming that preclude the pilot from taking actions that could place the aircraft outside the normal airframe design envelop. This was the result of a software design decision to protect the airframe by preventing the pilot from taking it outside its airframe design envelope.

Unlike the experience in the F-18 accidents described above, it is significantly better to rely on diverse technologies to permit excursions from desired design envelopes, as was done on the Boeing 777. In that design, both manual over-ride and mechanical backup systems were provided to ensure safe operation of the aircraft. The technology list above indicates that mechanical systems are generally considered more analyzable and predictable that software systems used for similar or the same function.

3.4.2 Undesirable Features. Some of the software used on the Therac-25 radiation therapy instrument is an example of the use of software that was used in a different manner than the original application's design [7]. One part of the software designed for use on the predecessor Therac-20 relied on a fuse to prevent improper radiation exposures. When the software was used on Therac-25, the fuse circuit was no longer present, allowing the Therac-25 to administer improper doses. This kind of use of a fuse was inadvertently an example of a relatively simple technology being used to protect against software commands that could cause dangerous doses of radiation to patients.

A fuse is generally considered more analyzable and predictable that a software system for protection purposes. As indicated in the technology list above, while software is a relatively complex technology for protecting against integrity failures, the fuse is a relatively simple and analyzable one.

3.4.3 Imperfect System Constructs. Imperfect system constructs are system hardware failures or software errors caused by something breaking at, or prior to, being exercised. Such a fault can spontaneously trigger a transition to a failure state, or such a transition can be triggered when a fault is exercised by a legitimate controller command. Unless the system is designed with sufficient fault tolerance, either of these failures can result in placing the system in a state from which it may not be able to recover. An example of such a failure is the Ariane 5 rocket accident [8]. In that system, the inertial platform software built and calibrated for the Ariane 4 was used in the Ariane 5 without recalibration. About 40 seconds after launch, when the software produced errors that the system could not handle, the Ariane 5 was intentionally destroyed, at significant financial loss.

The analyses of the Ariane 5 accident uncovered several places at which the software could have dealt with the hazard before it initiated a failure. These include the use of designed-in error trapping, the use of simulation testing, and the removal of the unit at launch time since it was not used after launch on the Ariane 5 .

In the above discussions, our classification partitions failures into (a) imperfect system functionality (the system can do more than intended), and (b) imperfect system constructs (the system can break). The F-18 is an example of a non-empty intersection of (a) and (b). The Therac-25 example given is an example of (a). The Ariane 5 is an example of a functionality (state space) mismatch between two system components -- thus it is an example of (a).

\section{Conclusions}

We have outlined a process for helping to ensure the integrity of systems that can experience failures from specific states. The process starts with a characterization of the system states with respect to their susceptibility to transitions to failure states. Such transitions can be 
evaluated for risk factors that indicate both the likelihood of such transitions and their consequences. In many cases, conventional approaches to estimation of probabilities of high consequence events are inadequate to deal with the likely consequences of their occurrence. This necessitates that we invoke techniques that are capable of demonstrating predictable safety. These techniques are outlined in a taxonomy that includes both technologies that are analyzable and predicable, and strategies for dealing with specific kinds of failures.

Design modifications must then be carefully scrutinized to ensure that they do not introduce additional functionality. This can be accomplished by modeling the integrity modifications as additional hybrid finite state machines.

\section{References}

[1] V. L. Winter, "A Synchronous Paradigm for Modeling Semi-Passive Reactive Systems," SAND98-1593, to be published.

[2] M. E. Ekman, P. W. Werner, J. M. Covan, and P. E. D'Antonio, "A Thematic Approach to System Safety,"
American Institute of Chemical Engineers, 32nd Loss Prevention Symposium, New Orleans, LA, March 11, 1998.

[3] W. R. Dunn, M. V. Frank, S. A. Epstein, and L. Doty, "Risk Assessment and Management of Safety-Critical, Digital Industrial Controls - Present Practices and Future Challenges," Proceedings of PSAM-II, Vol 1, March 1994.

[4] NUREG/CR-6303, Method for Performing Diversity and defense-in-Depth Analysis of Reactor Protection Systems, Lawrence Livermore National Laboratory.

[5] IEEE Standard 7-4.3.2 (1993).

[6] I. Peterson, Fatal Defect, Chasing Killer Computer Bugs, Vintage Books, 1995, p81.

[7] I. Peterson, Fatal Defect, Chasing Killer Computer Bugs, Vintage Books, 1995, p41.

[8], Butlin, Richard, "The Risks Digest," Wed, 5 Jun 96. [what about the Aviation Week article?] 


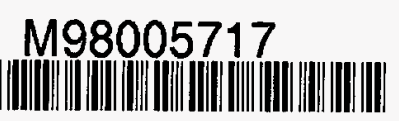

Report Number (14) $\frac{\sin D-9 \pi \cdot 0 \% \%}{\operatorname{sinf}-980809}$

Publ. Date (11)

Sponsor Code (18)

UC Category (19)

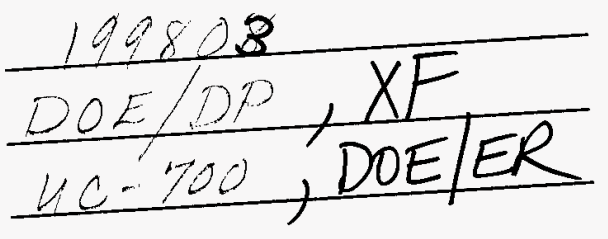

\section{5}

\section{DIIC QUALTTY INOPECTED I}

